ANNALES

POLONICI MATHEMATICI

$99.3(2010)$

\title{
On moduli spaces of semistable sheaves on Enriques surfaces
}

\author{
by MARCIN HAUZER (Warszawa)
}

\begin{abstract}
We describe some one-dimensional moduli spaces of rank 2 Gieseker semistable sheaves on an Enriques surface improving earlier results of H. Kim. In the case of a nodal Enriques surface the moduli spaces obtained are reducible for general polarizations. For unnodal Enriques surfaces we show how to reduce the study of moduli spaces of high even rank Gieseker semistable sheaves to low ranks. To prove this we use the method of K. Yoshioka who showed that in the odd rank case, one can reduce to rank 1 .
\end{abstract}

Introduction. An Enriques surface is a smooth projective surface $X$ satisfying the following conditions: the irregularity $q(X)=h^{1}\left(\mathscr{O}_{X}\right)$ is equal to 0 and the canonical line bundle $\omega_{X}$ is non-trivial but $\omega_{X}^{\otimes 2} \simeq \mathscr{O}_{X}$. For simplicity we assume that our surfaces are defined over an algebraically closed field $k$ of characteristic zero (otherwise we would have to change even the definition of an Enriques surface).

One of the aims of this note is to study geometry of one-dimensional moduli spaces of Gieseker semistable sheaves on Enriques surfaces. We are particularly interested in the case of rank 2 torsion free sheaves with the first Chern class equal to a half-pencil of an Enriques surface and with the (degree of) second Chern class equal to 1.

Before formulating our theorem let us recall that every Enriques surface is an elliptic fibration over $\mathbb{P}^{1}$ with two multiple fibres $2 F_{A}$ and $2 F_{B}$. $F_{A}$ and $F_{B}$ are reduced curves and they are called half-pencils.

THEOREM 0.1. There exists an explicit class of polarizations $H$ such that the moduli space $M_{X}\left(2, F_{A}, 1\right)$ of rank 2 Gieseker $H$-semistable sheaves with first Chern class $F_{A}$ and second Chern class 1 is isomorphic to $F_{B}$.

2010 Mathematics Subject Classification: Primary 14D20; Secondary 14J28.

Key words and phrases: moduli space, Enriques surface.

The author tragically died on 27 January 2010. The paper is a slightly edited version of one of the chapters of his un-submitted $\mathrm{PhD}$ thesis written under the supervision of Adrian Langer. 
This result corrects and strengthens the results of Chapter 5 of Kim's thesis (see [Kim1, Theorem 5.1]).

Let us recall that an Enriques surface is called unnodal if it does not contain any (-2)-curves. Kim considered only locally free sheaves on unnodal Enriques surfaces and claimed that the corresponding moduli space is non-reduced (which is false). Some parts of his arguments are also invalid without further assumptions on the polarization (e.g., in the proof of Kim1, Theorem 5.1] he changes the polarization and claims that the bundle remains stable).

It should be noted that in his later papers H. Kim claimed somewhat different results. In [Kim2, Example 1] he claimed a similar theorem for locally free sheaves and an arbitrary polarization (this statement is false). In his most recent paper [Kim3, II, Example] he claimed the result closest to Theorem 0.1 , the birationality of the moduli space $M_{X}\left(2, F_{A}, 1\right.$ ) (for an arbitrary polarization) with half-pencil $F_{B}$. In both cases no proof was provided.

The method of proof od Theorem 0.1 is quite similar to the one used by Okonek and Van de Ven $[\mathrm{OV}]$ in their computation of Donaldson invariants for Dolgachev surfaces (this result implied the existence of infinitely many homeomorphic surfaces which are not diffeomorphic). The main new ingredients are a good choice of polarizations and the method of description of singularities of moduli spaces (see Subsections 1.1 and 1.3).

One of the interests of this theorem stems from the interesting theorem proven by K. Yoshioka in [Yo, Theorem 4.6]. Namely, Yoshioka proved that for a general polarization the moduli space of semistable sheaves of odd rank and with a primitive Mukai vector on an unnodal Enriques surface is irreducible. On nodal Enriques surfaces Theorem 0.1 provides, for a general polarization, an example of a reducible moduli space of semistable sheaves of even rank and with a primitive Mukai vector.

In even rank for unnodal Enriques surfaces we have the following theorem:

TheOREM 0.2. Let $X$ be an unnodal Enriques surface and let $r$ be a positive even integer. Then for a general polarization the number of irreducible components of the moduli space of rank $r$ Gieseker semistable sheaves with fixed primitive Mukai vector and with fixed determinant is the same as the number of irreducible components of a similar moduli space for rank 2 or 4.

This theorem together with Yoshioka's results and Kim's conjecture in the rank 2 case suggests that on unnodal Enriques surfaces the moduli space of Gieseker semistable sheaves with fixed primitive Mukai vector and determinant should always be irreducible for a general polarization.

In fact we prove a much stronger form of Theorem 0.2 allowing one to compare virtual Hodge polynomials of some moduli spaces (see The- 
orem 2.8). Our proof follows the method of Yoshioka [Yo, Section 4] but the actual computations become more complicated than for odd rank. This method of proof also allows us to reprove the main result of [Kim1] (see Theorem 2.9.

The structure of the paper is as follows. In Section 1 we prove Theorem 0.1. Then in Section 2 we prove a refinement of Theorem 0.2. At the beginning of each section we describe the main steps in the proofs.

In the paper we use without warning the following facts about Enriques surfaces. If $X$ is an Enriques surface then $\chi\left(\mathscr{O}_{X}\right)=1$ and the Riemann-Roch theorem for a rank 2 vector bundle $E$ says that $\chi(E)=2+\frac{1}{2} c_{1}^{2}(E)-c_{2}(E)$. The canonical divisor of $X$ can be computed as $K_{X}=F_{A}-F_{B}=F_{B}-F_{A}$ (see [BHPV, VII.17]).

1. One-dimensional moduli spaces of semistable sheaves. In this section we prove Theorem 0.1. The structure of the proof is as follows. First we show how to choose polarizations for which Theorem 0.1 holds. Then we show that every 2 Gieseker $H$-semistable sheaf with first Chern class $F_{A}$ and second Chern class 1 can be obtained as a certain extension. This is used to prove that we have a set-theoretical bijection between the corresponding moduli scheme and a half-pencil $F_{B}$. The main difficulty is to prove that this is an isomorphism of schemes. To do so we study singularities of the moduli scheme. Then we construct some families of sheaves and use them to construct morphisms from the moduli scheme to the half-pencil and back providing the proof of Theorem 0.1 (see Theorem 1.14).

1.1. Choice of a polarization. In order to talk about the stability of sheaves on a surface $X$ we have to choose a polarization of $X$. Choosing it smartly we can exclude the existence of strictly semistable sheaves:

Lemma 1.1. There exists a polarization $H$ of $X$ such that all rank 2 Gieseker $H$-semistable sheaves $E$ with $c_{1} E=F_{A}$ and $c_{2} E=1$ are slope $H$-stable and there exists an ample divisor $L_{0}$ such that $H=L_{0}+n F_{A}$ for some integer $n>F_{A} . L_{0}$.

To prove this lemma we need to recall some results from [HL, Appendix $\mathrm{C}$ to Chapter 4].

Let $X$ be a smooth projective surface. Let $K^{+}$denote the positive cone of $X$, i.e., the set

$\left\{x \in \operatorname{Num}_{\mathbb{R}}(X): x^{2}>0\right.$ and $x . H>0$ for some ample divisior $\left.H\right\}$.

Let $\mathscr{H}$ denote the set of rays in $K^{+}$. This set can be identified with the hyperbolic manifold $\left\{H \in K^{+}:|H|=1\right\}$, where $|H|$ denotes $\left|H^{2}\right|^{1 / 2}$. We 
can define a hyperbolic metric on $\mathscr{H}$ by setting

for points $[H],\left[H^{\prime}\right] \in \mathscr{H}$.

$$
\beta\left([H],\left[H^{\prime}\right]\right)=\operatorname{arccosh}\left(\frac{H . H^{\prime}}{|H| \cdot\left|H^{\prime}\right|}\right)
$$

Definition 1.2. Let $r \geq 2$ and $\Delta>0$ be integers. A class $\xi \in \operatorname{Num}(X)$ is of type $(r, \Delta)$ if $-\left(r^{2} / 4\right) \Delta \leq \xi^{2}<0$. A wall defined by $\xi$ is the real 1-codimensional submanifold

$$
W_{\xi}=\{[H] \in \mathscr{H}: \xi . H=0\} \subset \mathscr{H} .
$$

LEMMA 1.3. Let us fix positive integers $r \geq 2$ and $\Delta$. Then the set of walls of type $(r, \Delta)$ is locally finite in $\mathscr{H}$.

THEOREM 1.4. Let $H$ be an ample divisor on $X$ and let $E$ be a slope $H$-semistable torsion free sheaf of rank $r$ and discriminant $\Delta$. Let $E^{\prime} \subset E$ be a subsheaf of rank $r^{\prime}, 0<r^{\prime}<r$, with $\mu_{H}\left(E^{\prime}\right)=\mu_{H}(E)$. Then the class $\xi:=r . c_{1}\left(E^{\prime}\right)-r^{\prime} . c_{1}(E)$ satisfies the following conditions:

$$
\xi . H=0 \quad \text { and } \quad-\frac{r^{2}}{4} \Delta \leq \xi^{2} \leq 0,
$$

and $\xi^{2}=0$ if and only if $\xi=0$. In particular, if $c_{1} \in \operatorname{Num}(X)$ is indivisible, and if $H$ is not on a wall of type $(r, \Delta)$, then a torsion free sheaf of rank $r$, with first Chern class $c_{1}$ and discriminant $\Delta$, is slope $H$-semistable if and only if it is slope $H$-stable.

Now we can prove Lemma 1.1.

Proof of Lemma 1.1. An Enriques surface $X$ viewed as an elliptic fibration $X \rightarrow \mathbb{P}^{1}$ always has a 2-section $G$ such that $G . F=2$ for general fibre (see [BHPV, Proposition VIII.17.5]). Therefore $G . F_{A}=1$ and $c_{1}=F_{A}$ is indivisible in $\operatorname{Num}(X)$. To make $M_{X}\left(2, F_{A}, 1\right)$ parameterize only stable sheaves we should choose a polarization which is not on a wall of type $(2,-4)$. We also need to work with a polarization which is close to the ray determined by $F_{A}$ in $\operatorname{Num}_{\mathbb{R}}(X)$ (and which is not ample). More precisely, the desired polarization should be of the form $L_{0}+n F_{A}$, where $L_{0}$ is an ample divisor and $n>F_{A}$. $L_{0}$. If we choose $L_{0}=L_{1}$ arbitrarily, it may happen that $L_{1}+n F_{A}$ is on a wall of type $(2,-4)$ for every $n$. In this case we fix $n_{0}>F_{A} \cdot L_{1}+1$ and we choose a vector $v \in \operatorname{Num}_{\mathbb{Q}}(X)$ such that

1. $L_{1}+v$ is an ample divisor,

2. $\left(L_{1}+v\right)+n_{0} F_{A}$ is not on a wall of type $(2,-4)$,

3. $v \cdot F_{A}<1$.

Such a vector $v$ exists because the cone of ample divisors is open and there exists a neighborhood of $L_{1}+n F_{A}$ which intersects only finitely many walls of type $(2,-4)$. Take $m \in \mathbb{N}$ such that $L_{1}^{\prime}:=m\left(L_{1}+v\right)$ belongs to $\operatorname{Num}(X)$. 
Then $L_{1}^{\prime}$ is ample and $L_{1}^{\prime}+m n_{0} F_{A}=m\left(L_{1}+v+n_{0} F_{A}\right)$ does not lie on any wall of type $(2,-4)$. Moreover, we have

$$
L_{1}^{\prime} \cdot F_{A}=m\left(L_{1}+v\right) \cdot F_{A}=m\left(L_{1} \cdot F_{A}+v \cdot F_{A}\right)<m\left(n_{0}-1+1\right)=m n_{0} .
$$

Therefore $L_{0}=L_{1}^{\prime}$ and $n=m n_{0}$ give a polarization $H=L_{0}+n F_{A}$ which is not on any wall of type $(2,-4)$ and such that $n>L_{0} \cdot F_{A}$. By Nakai's criterion the divisor $H$ is ample because $H^{2}=L_{0}^{2}+2 n L_{0} \cdot F_{A}+F_{A}^{2}=L_{0}^{2}+2 n L_{0} \cdot F_{A}>0$ and $H . D=L_{0} . D+n F_{A} . D>0$ since $F_{A} . D \geq 0$ for every effective divisor $D$. This finishes the proof.

1.2. Presentation of a sheaf as an extension. From now on we work only with polarizations described in Lemma 1.1 .

Lemma 1.5. Let $E$ be a rank 2 Gieseker $H$-semistable sheaf with the first Chern class $F_{A}$ and the second Chern class 1 . Then there exists a point $x \in X$ such that $E$ sits in a non-split exact sequence of the form

$$
0 \rightarrow \mathscr{O}_{X} \rightarrow E \rightarrow \mathscr{I}_{x} \otimes \mathscr{O}_{X}\left(F_{A}\right) \rightarrow 0 .
$$

Proof. By Lemma 1.1 we know that $E$ is slope $H$-stable. Since $H$ is fixed we will often omit $H$ when referring to stability of sheaves. By the Riemann-Roch theorem we have

$$
h^{0}(E)+h^{2}(E) \geq 2+\frac{1}{2} F_{A}^{2}-1=1 .
$$

By Serre duality we have $h^{2}(E)=\operatorname{dim} \operatorname{Hom}\left(E, \omega_{X}\right)=h^{0}\left(\omega_{X} \otimes E^{\vee}\right)$. Moreover, $\omega_{X} \otimes E^{\vee}$ is slope stable and locally free. However, $c_{1}\left(\omega_{X} \otimes E^{\vee}\right)=$ $c_{1}\left(E^{\vee}\right)=-F_{A}$ and $\mu\left(\omega_{X} \otimes E^{\vee}\right)<0$ so $\omega_{X} \otimes E^{\vee}$ has no global sections and $h^{2}(E)=0$. Therefore $h^{0}(E) \geq 1$ and $E$ fits in an exact sequence

$$
0 \rightarrow \mathscr{I}_{Z_{1}}(D) \rightarrow E \rightarrow \mathscr{I}_{Z_{2}} \otimes \mathscr{O}_{X}\left(-D+F_{A}\right) \rightarrow 0
$$

for some effective divisor $D$ and zero-dimensional subschemes $Z_{1}, Z_{2}$ of $X$ such that a section $\mathscr{O}_{X} \rightarrow E$ factors through $\mathscr{I}_{Z_{1}}(D) \rightarrow E$. By stability of $E$ we have

$$
\begin{aligned}
D \cdot H<\frac{1}{2} F_{A} \cdot H & \Leftrightarrow D \cdot\left(L_{0}+n F_{A}\right)<\frac{1}{2} F_{A} \cdot\left(L_{0}+n F_{A}\right) \\
& \Leftrightarrow D \cdot L_{0}+n F_{A} \cdot D<\frac{1}{2} F_{A} \cdot L_{0} .
\end{aligned}
$$

This implies that

$$
\left(F_{A} \cdot L_{0}\right)\left(F_{A} \cdot D\right)<n F_{A} \cdot D<\frac{1}{2} F_{A} \cdot L_{0} .
$$

Therefore $0 \leq F_{A} \cdot D<1 / 2$ and hence $F_{A} \cdot D=0$.

Now note that computation of the second Chern class from sequence (1) gives

$$
\text { D. }\left(F_{A}-D\right)+\operatorname{deg} Z_{1}+\operatorname{deg} Z_{2}=1 \text {. }
$$


So $D^{2}=\operatorname{deg} Z_{1}+\operatorname{deg} Z_{2}-1 \geq-1$. Since the intersection form on an Enriques surface is even, this implies that $D^{2} \geq 0$.

By stability of $E$ we also have $\left(F_{A}-2 D\right) . H>0$. So $F_{A}-2 D \neq 0, K_{X}$ and since $\left(F_{A}-2 D\right)^{2}=4 D^{2} \geq 0$ by [BHPV], Chapter VIII, Proposition 16.1.ii] we know that $\left|F_{A}-2 D\right| \neq \emptyset$. Therefore there exists an effective divisor $C$ such that $F_{A} \sim 2 D+C$. But $h^{0}\left(\mathscr{O}_{X}\left(F_{A}\right)\right)=1$ (see the proof of Lemma 17.3 in [BHPV, Chapter VIII]) and hence we have equality: $F_{A}=2 D+C$. The half-pencil $F_{A}$ has no multiple components, so the only possibility is that $D=0$.

Now existence of the morphism $\mathscr{O}_{X} \rightarrow \mathscr{I}_{Z_{1}}$ shows that $Z_{1}$ must be empty. This allows us to compute the length of $Z_{2}$ :

$$
1=c_{2}(E)=c_{2}\left(\mathscr{I}_{Z} \otimes \mathscr{O}_{X}\left(-D+F_{A}\right)\right)+D \cdot\left(-D+F_{A}\right)=l\left(Z_{2}\right) .
$$

Splitting of sequence (1) would contradict the stability of $E$, so the sequence is non-split.

Sheaves appearing as extensions of the form as in the previous lemma are characterized by the following lemma:

Lemma 1.6. Let $E$ be a sheaf given by a non-trivial extension

$$
0 \rightarrow \mathscr{O}_{X} \rightarrow E \rightarrow \mathscr{I}_{x} \otimes \mathscr{O}_{X}\left(F_{A}\right) \rightarrow 0
$$

for some closed point $x \in X$. Then $x \in F_{B}, E$ is locally free and it is uniquely determined by $x$.

Proof. Extensions of $\mathscr{I}_{x} \otimes \mathscr{O}_{X}\left(F_{A}\right)$ by $\mathscr{O}_{X}$ are parameterized by the group $\operatorname{Ext}^{1}\left(\mathscr{I}_{x} \otimes \mathscr{O}_{X}\left(F_{A}\right), \mathscr{O}_{X}\right)$. By Serre duality this group is dual to $\operatorname{Ext}^{1}\left(\omega_{X}, \mathscr{I}_{x} \otimes \mathscr{O}_{X}\left(F_{A}\right)\right)=H^{1}\left(X, \mathscr{I}_{x} \otimes \mathscr{O}_{X}\left(F_{A}\right) \otimes \omega_{X}\right)=H^{1}(X$, $\left.\mathscr{I}_{X} \otimes \mathscr{O}_{X}\left(F_{B}\right)\right)$ because $\omega_{X} \simeq \mathscr{O}_{X}\left(F_{B}-F_{A}\right)$. Consider an exact sequence

$$
0 \rightarrow \mathscr{I}_{x} \rightarrow \mathscr{O}_{X} \rightarrow \mathscr{O}_{x} \rightarrow 0
$$

and tensorize it with $\mathscr{O}_{X}\left(F_{B}\right)$. By proof of Lemma 17.3 in BHPV, Chapter VIII] we have $h^{0}\left(\mathscr{O}_{X}\left(F_{B}\right)\right)=1$ and $h^{1}\left(\mathscr{O}_{X}\left(F_{B}\right)\right)=0$. Thus we have the following long exact sequence:

$$
\begin{aligned}
0 \rightarrow H^{0}\left(\mathscr{I}_{x} \otimes \mathscr{O}_{X}\left(F_{B}\right)\right) & \rightarrow H^{0}\left(\mathscr{O}_{X}\left(F_{B}\right)\right) \\
& \rightarrow H^{0}\left(\mathscr{O}_{x}\left(F_{B}\right)\right) \rightarrow H^{1}\left(\mathscr{I}_{x} \otimes \mathscr{O}_{X}\left(F_{B}\right) \rightarrow 0 .\right.
\end{aligned}
$$

Since $h^{0}\left(\mathscr{O}_{x}\left(F_{B}\right)\right)=1, H^{1}\left(\mathscr{I}_{x} \otimes \mathscr{O}_{X}\left(F_{B}\right)\right)$ has dimension 0 or 1 . But by assumption $E$ comes from a non-trivial extension, so $h^{1}\left(\mathscr{I}_{x} \otimes \mathscr{O}_{X}\left(F_{B}\right)\right)=1$. This implies that $h^{0}\left(\mathscr{I}_{x} \otimes \mathscr{O}_{X}\left(F_{B}\right)\right)=1$ and therefore $x$ lies in the zero set of a non-trivial section of $\mathscr{O}_{X}\left(F_{B}\right)$, i.e., $x \in F_{B}$. The local freeness of $E$ follows from the Cayley-Bacharach property of a single point $x \in F_{B}$ with respect to the linear system $\left|F_{B}\right|=\left\{F_{B}\right\}$. 
Lemma 1.7. Let $E$ be as in the previous lemma. Then $E$ is slope $H$ stable.

Proof. To check the stability of $E$ it is sufficient to consider subsheaves of the form $\mathscr{O}_{X}(C) \subset E$. We can also assume that this subsheaf is saturated, i.e., the quotient $E / \mathscr{O}_{X}(C)$ is torsion free. If the linear system $|-C|$ is nonempty then $C . H<0 \leq \frac{1}{2} F_{A} . H$. So we can assume that $|-C|=\emptyset$. Using the short exact sequence

$$
0 \rightarrow \mathscr{O}_{X}(-C) \rightarrow E(-C) \rightarrow \mathscr{I}_{x}\left(F_{A}-C\right) \rightarrow 0
$$

and the fact that $h^{0}(E(-C))>0$ we get $h^{0}\left(\mathscr{I}_{x}\left(F_{A}-C\right)\right)>0$. Therefore there exists an effective divisor $R \sim F_{A}-C$ which passes through $x$. If $R . F_{A} \geq 1$ then

$$
\begin{aligned}
C . H & =\left(L_{0}+n F_{A}\right) \cdot\left(F_{A}-R\right)=L_{0} \cdot F_{A}-n R \cdot F_{A}-R \cdot L_{0} \\
& \leq L_{0} \cdot F_{A}-n<0 \leq \frac{1}{2} F_{A} \cdot H .
\end{aligned}
$$

Therefore we can assume that $R \cdot F_{A}=0$, which implies that $C \cdot F_{A}=0$.

Now note that by assumption there exists a zero-dimensional subscheme $Z$ such that $E$ sits in a short exact sequence of the form

$$
0 \rightarrow \mathscr{O}_{X}(C) \rightarrow E \rightarrow \mathscr{I}_{Z} \otimes \mathscr{O}_{X}(R) \rightarrow 0 .
$$

Computing the second Chern class we get $C R+\operatorname{deg} Z=1$. Therefore $-R^{2}=$ $C R \leq 1$, which implies that $R^{2} \geq 0$ and $C R \leq 0$. But this implies that $C^{2}=-C R \geq 0$, so by [BHPV, Proposition VIII.16.1] the linear system $|C|$ is non-empty. Therefore $F_{A}=C+R$, which contradicts the fact that $x$ lies on $R$.

Summarizing we have the following corollary:

COROLlary 1.8. There exists a bijection between closed points of $M_{X}\left(2,1, F_{A}\right)$ and $F_{B}$.

Proof. The only fact that remains to be proved is that for a sheaf $E$, a point $x \in F_{B}$ such that we have a non-split exact sequence of the form

$$
0 \rightarrow \mathscr{O}_{X} \rightarrow E \rightarrow \mathscr{I}_{x} \otimes \mathscr{O}_{X}\left(F_{A}\right) \rightarrow 0
$$

is uniquely determined. To prove this, note that $H^{0}\left(\mathscr{I}_{x} \otimes \mathscr{O}_{X}\left(F_{A}\right)\right)=0$, since $x$ does not lie on $F_{A}$. Therefore $H^{0}(E)$ is one-dimensional and $x$ is the zero set of the unique (up to a scalar multiple) non-trivial section of $E$.

1.3. Singularities of $M_{X}\left(2,1, F_{A}\right)$. In order to analyze the smoothness of $M_{X}\left(2,1, F_{A}\right)$ we have to consider $\operatorname{Ext}^{2}(E, E)=\left(\operatorname{Hom}\left(E, E \otimes \omega_{X}\right)\right)^{*}$. Since $E$ is slope stable and of the same slope as $E\left(K_{X}\right)$ every non-zero homomorphism $s \in \operatorname{Hom}\left(E, E \otimes \omega_{X}\right)$ gives rise to an isomorphism. Hence $\operatorname{Ext}^{2}(E, E)$ vanishes if and only if $E$ and $E\left(K_{X}\right)$ are not isomorphic. Both 
$E$ and $E\left(K_{X}\right)$ represent points in $M_{X}\left(2, F_{A}, 1\right)$ so we can present them as extensions:

$$
\begin{aligned}
0 \rightarrow \mathscr{O}_{X} \rightarrow E & \rightarrow \mathscr{I}_{x_{0}} \otimes \mathscr{O}_{X}\left(F_{A}\right) \rightarrow 0, \\
0 \rightarrow \mathscr{O}_{X} \rightarrow E\left(K_{X}\right) & \rightarrow \mathscr{I}_{x_{1}} \otimes \mathscr{O}_{X}\left(F_{A}\right) \rightarrow 0,
\end{aligned}
$$

for some uniquely determined $x_{0}, x_{1} \in F_{B}$. In particular, $E$ and $E\left(K_{X}\right)$ are isomorphic if and only if $x_{0}=x_{1}$. Now we need the following lemma:

Lemma 1.9. Let $\mathfrak{m}_{x, F_{B}}$ denote the ideal sheaf of a point $x$ in $F_{B}$. Then the sheaves $\mathfrak{m}_{x_{1}, F_{B}}$ and $\mathfrak{m}_{x_{0}, F_{B}} \otimes \mathscr{O}_{F_{B}}\left(F_{B}\right)$ are isomorphic as $\mathscr{O}_{F_{B}}$-modules.

Proof. Let us consider the following commutative diagram:

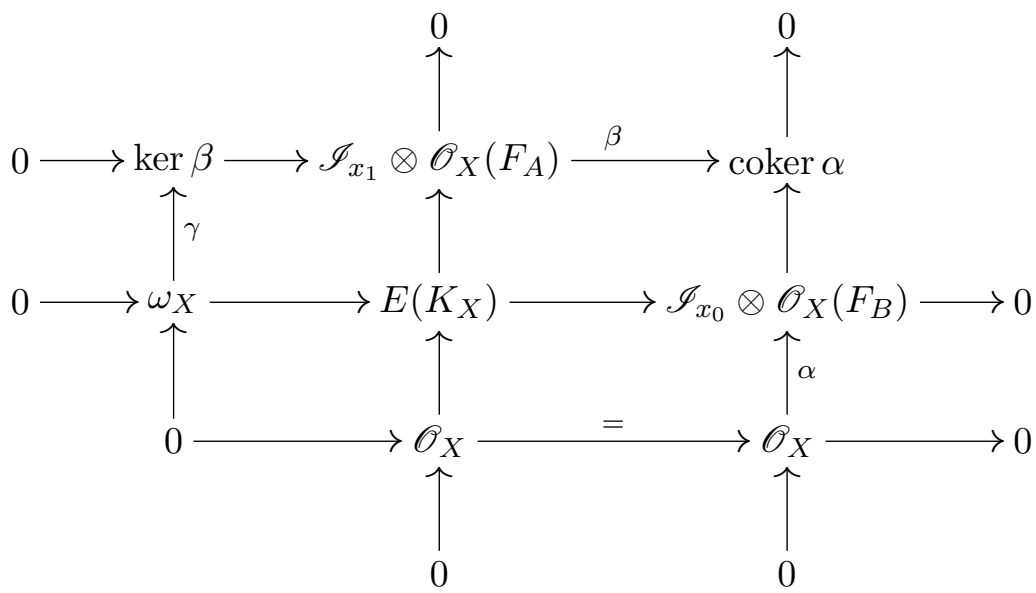

where the middle horizontal sequence is obtained from (2) by multiplying by $\omega_{X}$ and using $K_{X}=F_{B}-F_{A}$. Note that $\gamma$ in this diagram must be an isomorphism and $\beta$ must be surjective. In particular, we have a short exact sequence

$$
0 \rightarrow \omega_{X} \longrightarrow \mathscr{I}_{x_{1}} \otimes \mathscr{O}_{X}\left(F_{A}\right) \longrightarrow \operatorname{coker} \alpha \rightarrow 0 .
$$

By definition we also have an isomorphism coker $\alpha \simeq \mathfrak{m}_{x_{0}, F_{B}} \otimes \mathscr{O}_{F_{B}}\left(F_{B}\right)$. On the other hand the above exact sequence implies that coker $\alpha \simeq \mathfrak{m}_{x_{1}, F_{B}} \otimes$ $\mathscr{O}_{F_{B}}\left(F_{A}\right) \simeq \mathfrak{m}_{x_{1}, F_{B}}$, which proves the required assertion.

Proposition 1.10. Let $E$ and $E(K)$ be determined by $x_{0}, x_{1} \in F_{B}$, respectively.

1. If $x_{0}$ is a smooth point of $F_{B}$ then $x_{1}$ is also a smooth point of $F_{B}$ and it is the zero set of the unique (up to a scalar) section of $\mathscr{O}_{F_{B}}\left(F_{B}+x_{0}\right)$. In particular, $x_{1} \neq x_{0}$.

2. If $x_{0}$ is a singular point of $F_{B}$ then $x_{1}=x_{0}$.

Proof. Let us first recall that by [CD, Theorem 5.7.5] the half-pencils $F_{A}$ and $F_{B}$ have only nodal singularities. Let $C$ be a curve with a nodal 
singularity at $x$. Then $m_{x, C}$ is not a line bundle. Otherwise, the maximal ideal of the completion $\hat{\mathscr{O}}_{C, x} \simeq k[[a, b]] /(a b)$ of the local ring of $C$ at $x$ would be generated by one element. But this is not possible.

Let us now assume that $x_{0}$ is a smooth point of $F_{B}$. Then $\mathfrak{m}_{x_{0}, F_{B}} \simeq$ $\mathscr{O}_{F_{B}}\left(-x_{0}\right)$. Therefore by the above lemma $\mathfrak{m}_{x_{1}, F_{B}} \simeq \mathscr{O}_{F_{B}}\left(F_{B}-x_{0}\right)$ is a line bundle, which implies that $x_{1}$ is a smooth point of $F_{B}$ and $\mathscr{O}_{F_{B}}\left(x_{1}\right) \simeq$ $\mathscr{O}_{F_{B}}\left(F_{B}+x_{0}\right)$ (we use the fact that $\mathscr{O}_{F_{B}}\left(2 F_{B}\right)$ is a trivial line bundle). From the short exact sequence

$$
0 \rightarrow \mathscr{O}_{X} \rightarrow \mathscr{O}_{X}\left(F_{B}\right) \rightarrow \mathscr{O}_{F_{B}}\left(F_{B}\right) \rightarrow 0
$$

we see that $h^{0}\left(\mathscr{O}_{F_{B}}\left(F_{B}\right)\right)=0$ (in particular $\mathscr{O}_{F_{B}}\left(F_{B}\right) \simeq \mathscr{O}_{F_{B}}\left(x_{1}-x_{0}\right)$ is non-trivial and hence $\left.x_{1} \neq x_{0}\right)$. So from the short exact sequence

$$
0 \rightarrow \mathscr{O}_{F_{B}}\left(F_{B}\right) \rightarrow \mathscr{O}_{F_{B}}\left(F_{B}+x_{0}\right) \rightarrow \mathscr{O}_{x_{0}}\left(F_{B}+x_{0}\right) \simeq \mathscr{O}_{x_{0}} \rightarrow 0
$$

we see that $h^{0}\left(\mathscr{O}_{F_{B}}\left(F_{B}+x_{0}\right)\right)=1$, which proves the first part of the proposition.

To prove the second part let us assume that $x_{0}$ is a singular point of $F_{B}$. Then $x_{1}$ is also a singular point of $F_{B}$, since $\mathfrak{m}_{x_{1}, F_{B}}$ is not a line bundle. In particular, if $F_{B}$ is irreducible then $x_{1}=x_{0}$. So we can assume that $F_{B}$ is reducible. In this case all irreducible components of $F_{B}$ are smooth. Let $C$ be an irreducible component of $F_{B}$ containing $x_{0}$. Then we claim that $\mathfrak{m}_{x_{0}, F_{B}} \otimes \mathscr{O}_{C} \simeq \mathscr{O}_{C}\left(-x_{0}\right) \oplus \mathscr{O}_{x_{0}}$. To prove this note that we have a canonical surjection $\mathfrak{m}_{x_{0}, F_{B}} \rightarrow \mathfrak{m}_{x_{0}, C}=\mathscr{O}_{C}\left(-x_{0}\right)$. Tensoring it by $\mathscr{O}_{C}$ we need to prove that the kernel is isomorphic to the sheaf $\mathscr{O}_{x_{0}}$. We can do it locally passing to local completions at the maximal ideal of $\mathscr{O}_{C, x}$. Then the above map looks like the map

$$
(a, b) \otimes_{k[[a, b]] /(a b)} k[[a, b]] /(a) \rightarrow(a, b) \cdot k[[a, b]] /(a) \simeq b k[[b]]
$$

and the kernel of this map is generated by $a \otimes 1$, which proves our claim.

The above claim implies that $\mathfrak{m}_{x_{1}, F_{B}} \otimes \mathscr{O}_{C}$ contains torsion, which is possible only if $x_{1}$ lies on $C$. But this implies that $x_{1}$ lies on the same irreducible component of $F_{B}$ as $x_{0}$ and hence $x_{1}=x_{0}$.

The above proposition implies the following corollary:

Corollary 1.11. Let $[E] \in M_{X}\left(2, F_{A}, 1\right)$. Then $E \simeq E(K)$ if and only if the point $x_{0}$ associated to $E$ is a singular point of $F_{B}$.

1.4. Family of sheaves. In order to obtain a morphism from $F_{B}$ to $M_{X}\left(2,1, F_{A}\right)$ we have to construct a family of sheaves $\mathscr{E}$ on $F_{B} \times X$ such that for every $x \in F_{B}$ the sheaf $\left[\mathscr{E}_{\mid\{x\} \times X}\right] \in M_{X}\left(2,1, F_{A}\right)$. Obviously, we will try to do it in such a way that $\mathscr{E}_{\mid\{x\} \times X}$ corresponds to the non-trivial extension of $\mathscr{I}_{x} \otimes \mathscr{O}_{X}\left(F_{A}\right)$ by $\mathscr{O}_{X}$. 
Let $\Gamma$ denote the graph in the product $F_{B} \times X$ of the inclusion $F_{B} \subset X$ and $\mathscr{I}_{\Gamma}$ be its ideal sheaf. Let $\pi_{i}$ denote the projection from $F_{B} \times X$ on the $i$ th factor. Observe that, since $\operatorname{Ext}^{1}\left(\mathscr{I}_{x} \otimes \mathscr{O}_{X}\left(F_{A}\right), \mathscr{O}_{X}\right)$ is one-dimensional for every $x \in F_{B}$, the sheaf

$$
\mathscr{L}=\underline{\operatorname{Ext}}_{\pi_{1}}^{1}\left(\mathscr{I}_{\Gamma} \otimes \pi_{2}^{*}\left(\mathscr{O}_{X}\left(F_{A}\right)\right), \mathscr{O}_{F_{B} \times X}\right)
$$

is a line bundle on $F_{B}$. By [BPS, p.137] there is a spectral sequence $H^{p}\left(\underline{\operatorname{Ext}}_{\pi_{1}}^{q}\left(\mathscr{I}_{\Gamma} \otimes \pi_{2}^{*} \mathscr{O}_{X}\left(F_{A}\right), \pi_{1}^{*}\left(\mathscr{L}^{\vee}\right)\right)\right) \rightarrow \operatorname{Ext}^{p+q}\left(\mathscr{I}_{\Gamma} \otimes \pi_{2}^{*} \mathscr{O}_{X}\left(F_{A}\right), \pi_{1}^{*}\left(\mathscr{L}^{\vee}\right)\right)$, which gives a long exact sequence

$$
\begin{aligned}
& 0 \rightarrow H^{1}\left(\underline{\operatorname{Hom}}_{\pi_{1}}\left(\mathscr{I}_{\Gamma} \otimes \pi_{2}^{*} \mathscr{O}_{X}\left(F_{A}\right), \pi_{1}^{*}\left(\mathscr{L}^{\vee}\right)\right)\right) \\
& \rightarrow \operatorname{Ext}^{1}\left(\mathscr{I}_{\Gamma} \otimes \pi_{2}^{*} \mathscr{O}_{X}\left(F_{A}\right), \pi_{1}^{*}\left(\mathscr{L}^{\vee}\right)\right) \rightarrow H^{0}\left(\underline{\operatorname{Ext}}_{\pi_{1}}^{1}\left(\mathscr{I}_{\Gamma} \otimes \pi_{2}^{*} \mathscr{O}_{X}\left(F_{A}\right), \pi_{1}^{*}\left(\mathscr{L}^{\vee}\right)\right)\right) \\
& \rightarrow H^{2}\left(\underline{\operatorname{Hom}}_{\pi_{1}}\left(\mathscr{I}_{\Gamma} \otimes \pi_{2}^{*} \mathscr{O}_{X}\left(F_{A}\right), \pi_{1}^{*}\left(\mathscr{L}^{\vee}\right)\right)\right) .
\end{aligned}
$$

For a fixed $x \in F_{B}$ we have $\operatorname{Hom}\left(\mathscr{I}_{x} \otimes \mathscr{O}_{X}\left(F_{A}\right), \mathscr{O}_{X}\right)=0$. Hence $\underline{\operatorname{Hom}}_{\pi_{1}}\left(\mathscr{I}_{\Gamma} \otimes \pi_{2}^{*} \mathscr{O}_{X}\left(F_{A}\right), \pi_{1}^{*}\left(\mathscr{L}^{\vee}\right)\right)=0$. The isomorphism

$$
\begin{aligned}
\operatorname{Ext}^{1}\left(\mathscr{I}_{\Gamma} \otimes \pi_{2}^{*} \mathscr{O}_{X}\left(F_{A}\right), \pi_{1}^{*}\left(\mathscr{L}^{\vee}\right)\right) & \simeq H^{0}\left(\underline{\operatorname{Ext}}_{\pi_{1}}^{1}\left(\mathscr{I}_{\Gamma} \otimes \pi_{2}^{*} \mathscr{O}_{X}\left(F_{A}\right), \pi_{1}^{*}\left(\mathscr{L}^{\vee}\right)\right)\right) \\
& \simeq H^{0}\left(\mathscr{L} \otimes \mathscr{L}^{\vee}\right) \simeq H^{0}\left(\mathscr{O}_{F_{B}}\right)
\end{aligned}
$$

shows that with $1 \in H^{0}\left(\mathscr{O}_{F_{B}}\right)$ we can naturally associate an extension

$$
0 \rightarrow \mathscr{O}_{F_{B} \times X} \rightarrow \mathscr{E} \rightarrow \mathscr{I}_{\Gamma} \otimes \pi_{2}^{*} \mathscr{O}_{X}\left(F_{A}\right) \otimes \pi_{1}^{*}(\mathscr{L}) \rightarrow 0
$$

on $F_{B} \times X$, where $\mathscr{E}$ is locally free and after restricting to $\{x\} \times X$ gives the sheaf associated to $x$. The sheaf $\mathscr{E}$ can be regarded as a family of sheaves parameterized by $F_{B}$. Therefore we get a morphism $F_{B} \rightarrow M_{X}\left(2, F_{A}, 1\right)$.

COROLlary 1.12. The moduli space $M_{X}\left(2, F_{A}, 1\right)$ is a connected reduced curve.

Proof. By Lemma 1.8 the morphism $F_{B} \rightarrow M_{X}\left(2, F_{A}, 1\right)$ constructed above is bijective on closed points.

If $[E] \in M_{X}\left(2, F_{A}, 1\right)$ corresponds to a smooth point of $F_{B}$ then by Corollary 1.11 we have $E \Varangle E(K)$ and therefore $\operatorname{Ext}^{2}(E, E)=0$. By [La, Corollary 5.1.2], $[E]$ is a smooth point and the dimension of $M_{X}\left(2, F_{A}, 1\right)$ at this point is equal to $\operatorname{dim} \operatorname{Ext}^{1}(E, E)=1+c_{2}\left(E \otimes E^{\vee}\right)-4 \chi\left(\mathscr{O}_{X}\right)=1$. Therefore $M_{X}\left(2, F_{A}, 1\right)$ is connected and reduced at every generic point and it has a finite number of singular points corresponding to singularities of $F_{B}$.

Note that the expected dimension of the moduli space $M_{X}\left(2, F_{A}, 1\right)$ at any point $[E]$ is equal to $\operatorname{dim} \operatorname{Ext}^{1}(E, E)-\operatorname{dim} \operatorname{Ext}^{2}(E, E)=1$. Therefore by [HL, Theorem 4.5.8] the moduli space $M_{X}\left(2, F_{A}, 1\right)$ is a locally complete intersection. Since $M_{X}\left(2, F_{A}, 1\right)$ is reduced at every generic point it is reduced everywhere. 
We can also construct a morphism in the opposite direction.

By [HL, Theorem 4.6.5] the moduli space $M_{X}\left(2, F_{A}, 1\right)$ is a fine moduli space. Indeed, the chosen polarization excludes the existence of strictly semistable sheaves and if $[E] \in M_{X}\left(2, F_{A}, 1\right)$ then $\chi(E)=1$ (so we can take $B=\mathscr{O}_{X}$ in the above mentioned theorem). Let $\mathscr{F}$ be a universal family on $M_{X}\left(2, F_{A}, 1\right) \times X$ and let $p_{1}, p_{2}$ denote the projections on the first and the second factor, respectively. For every closed point $[E] \in M_{X}\left(2, F_{A}, 1\right)$ there exists an extension

$$
0 \rightarrow \mathscr{O}_{X} \rightarrow E \rightarrow \mathscr{I}_{x} \otimes \mathscr{O}_{X}\left(F_{A}\right) \rightarrow 0
$$

for some $x \in F_{B}$. Hence the long exact sequence of cohomology gives $H^{0}\left(\mathscr{O}_{X}\right) \simeq H^{0}(E)$. Moreover, we have already proved that $h^{2}(E)=0$ so the equality $\chi(E)=1$ gives us the vanishing of $H^{1}(E)$. The following theorem shows that $p_{1_{*}} \mathscr{F}$ is an invertible sheaf on $M_{X}\left(2, F_{A}, 1\right)$ :

Theorem 1.13 ([EGA, Theorem 7.9.9]). Let $Y$ be a locally noetherian scheme, $f: X \rightarrow Y$ a proper morphism, $\mathscr{F}$ a sheaf of $\mathscr{O}_{X}$-modules flat over $Y$. Assume that there exists $i_{0} \in \mathbb{Z}$ that $h^{i}\left(f^{-1}(y), \mathscr{F} \otimes_{\mathscr{O}_{u}} k(y)\right)=0$ for every $i \neq i_{0}$ and every $y \in Y$. Then $R^{i_{0}} f_{*} \mathscr{F}$ is locally free at $y$ and its rank is equal to $h^{i_{0}}\left(f^{-1}(y), \mathscr{F} \otimes_{\mathscr{O}_{u}} k(y)\right)$.

Moreover, $\operatorname{Hom}\left(p_{1}{ }^{*} p_{1 *} \mathscr{F}, \mathscr{F}\right)=\operatorname{Hom}\left(p_{1 *} \mathscr{F}, p_{1 *} \mathscr{F}\right)$ so we can consider the map

$$
p_{1}{ }^{*} p_{1 *} \mathscr{F} \rightarrow \mathscr{F}
$$

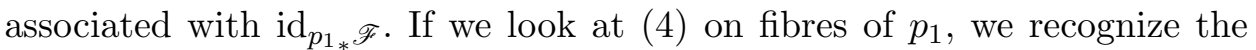
extension from Lemma 1.6. So the cokernel of (4) is isomorphic to $\mathscr{I}_{C} \otimes$ $p_{2}^{*} \mathscr{O}_{X}\left(F_{A}\right) \otimes p_{1}^{*} \mathscr{L}^{\prime}$ for some invertible sheaf $\mathscr{L}^{\prime}$ on $M_{X}\left(2, F_{A}, 1\right)$ and a curve $C \subset M_{X}\left(2, F_{A}, 1\right) \times X$. Note that by restricting $C$ to $[E] \times X$ we get a point $x \in X$ determining $E$. The sheaf $\mathscr{I}_{C}$ gives us a sheaf $\mathscr{O}_{C}$ which can be treated as a family of zero-dimensional subschemes of $X$ parameterized by $M_{X}\left(2, F_{A}, 1\right)$. This gives a morphism $M_{X}\left(2, F_{A}, 1\right) \rightarrow \operatorname{Hilb}^{1}(X) \simeq X$ which factors through $F_{B}$.

THEOREM 1.14. Let us fix a polarization $H$ satisfying the conditions from Lemma 1.1. Then the moduli space $M_{X}\left(2, F_{A}, 1\right)$ of rank 2 Gieseker $H$-semistable sheaves with first Chern class $F_{A}$ and second Chern class 1 is isomorphic to $F_{B}$.

Proof. We have already constructed morphisms $M_{X}\left(2, F_{A}, 1\right) \rightarrow F_{B}$ and $F_{B} \rightarrow M_{X}\left(2, F_{A}, 1\right)$ which give identity on closed points when they are composed. Since both schemes are reduced these morphisms are isomorphisms.

2. Moduli spaces of Gieseker semistable sheaves of even rank. In this section we prove Theorem 0.2 . First we prove some simple results 
about lattices. Then we recall some results on the Mukai lattice for an Enriques surface and we prove some lemmas concerning this lattice. Finally we use these results and Yoshioka's method to prove a refinement of Theorem 0.2 (see Theorem 2.8).

2.1. Some simple results on lattices. Let $L$ be a finitely generated free $\mathbb{Z}$-module. An element $x \in L$ is called primitive if the quotient module $L / \mathbb{Z} x$ is torsion free. A lattice is a pair consisting of a finitely generated free $\mathbb{Z}$-module and an integral bilinear (in our case also symmetric) form $\langle\cdot, \cdot\rangle$.

In the following, $-E_{8}$ denotes the lattice $\mathbb{Z}^{8}$ with canonical basis $\left\{e_{1}, \ldots, e_{8}\right\}$ whose intersection matrix $\left(\left\langle e_{i}, e_{j}\right\rangle\right)$ is the negative of the Cartan matrix of the root system $E_{8}$.

Lemma 2.1. Let $L$ be a finitely generated free $\mathbb{Z}$-module of rank $\operatorname{rk} L=$ $n>1$. Let $r$ be a positive integer and let $x$ be an element of $L$. Let us set $l=$ $\operatorname{gcd}(r, x)$. Then there exists $\xi \in L$ such that $(x+r \xi) / l$ is a primitive element in L. Moreover, if there exists a bilinear form $\langle\cdot, \cdot\rangle$ such that $(L,\langle\cdot, \cdot\rangle)$ is isometric to $-E_{8}$ then for an arbitrary number $M$ we can choose $\xi$ such that $2\langle x, \xi\rangle+r\langle\xi, \xi\rangle<M$.

Proof. Let $\left\{e_{1}, \ldots, e_{n}\right\}$ be a basis for $L$. If $x=0$ then as $\xi$ we can take an arbitrary primitive element in $L$. If $x \neq 0$ then we can assume that $x / l=\sum a_{i} e_{i}$ with $a_{1} \neq 0$. Let $k$ be the product of all prime numbers which divide $a_{1}$ but do not divide $a_{2}$. We claim that for all $b$ such that $\operatorname{gcd}\left(b, a_{1}\right)=1$ the element $y:=(r / l) k b e_{2}+x / l$ is primitive. In our chosen basis, $y$ has coordinates $\left(a_{1},(r / l) k b+a_{2}, \ldots, a_{n}\right)$. Let $p$ be a prime divisor of $a_{1}$. Then either $p \mid a_{m}$ for all $m$ or there exists $m$ such that $p \nmid a_{m}$. In the first case $p \nmid r / l$ because otherwise $\operatorname{gcd}(x, r)>l$. Then $p$ divides neither $k$ nor $b$. Therefore $p \nmid \operatorname{gcd}\left(a_{1},(r / l) k b+a_{2}\right)$ and hence $p \nmid y$.

Now consider the case when there exists $m$ such that $p \nmid a_{m}$. If $m \neq 2$ then $p \nmid \operatorname{gcd}\left(a_{1}, a_{m}\right)$. If $m=2$ then $p \mid k$ and $p \nmid \operatorname{gcd}\left(a_{1},(r / l) k b+a_{2}\right)$.

To finish the proof for the lattice $-E_{8}$ we may assume that $\left\langle e_{i}, e_{i}\right\rangle=-2$ and $\left\langle e_{2}, e_{3}\right\rangle=0$. If $x=0$ then we take $\xi=p e_{2}+q e_{3}$ for prime numbers $p, q \gg 0$. In the other case it is enough to notice that $2\left\langle x, k b e_{2}\right\rangle+r\left\langle k b e_{2}, k b e_{2}\right\rangle$ is a quadratic polynomial in $b$ with negative leading coefficient so for $b \gg 0$ it is less than $M$.

For the convenience of the reader we include the proof of the following well known lemma.

LEMma 2.2. Let $(L,\langle\rangle$,$) be a unimodular lattice of rank n$ and $y \in L$ be a primitive element. Then for every $m \in \mathbb{Z}$ there exists $\eta \in L$ such that $\langle\eta, y\rangle=m$.

Proof. It is enough to prove the lemma for $m=1$. Let $M$ be the sublattice of $L$ generated by $y$. Then $L / M$ is a free $\mathbb{Z}$-module with basis 
$\left[\alpha_{1}\right], \ldots,\left[\alpha_{n-1}\right]$. Then $\alpha_{1}, \ldots, \alpha_{n-1}, \alpha_{n}=y$ is a basis for $L$. The determinant of the matrix $\left(\left\langle\alpha_{i}, \alpha_{j}\right\rangle\right)$ is equal to \pm 1 so

$$
\operatorname{gcd}\left(\left\langle\alpha_{1}, \alpha_{n}\right\rangle, \ldots,\left\langle\alpha_{i}, \alpha_{n}\right\rangle, \ldots,\left\langle\alpha_{n}, \alpha_{n}\right\rangle\right)=1 .
$$

Therefore there exist integers $a_{i}$ such that $\sum a_{i}\left\langle\alpha_{i}, \alpha_{n}\right\rangle=1$ and we can take $\eta=\sum a_{i} \alpha_{i}$.

2.2. Mukai's lattice of an Enriques surface. Let $X$ be a complex Enriques surface and let $K(X)$ be the Grothendieck group of $X$. Any class in $K(X)$ has well defined Chern classes. The Mukai vector $v(x)$ of a class $x \in K(X)$ is defined as the following element of $H^{2 *}(X, \mathbb{Q})$ :

$$
v(x):=\operatorname{ch}(x) \sqrt{\operatorname{td}_{X}}=r k(x)+c_{1}(x)+\left(\frac{\operatorname{rk}(x)}{2} \varrho_{X}+\operatorname{ch}_{2}(x)\right) \in H^{2 *}(X, \mathbb{Q}),
$$

where $\rho_{X}$ is the fundamental class of $X$ (i.e., a class in $H^{4}(X, \mathbb{Q})$ such that $\left.\int_{X} \rho_{X}=1\right)$. The induced map $v: K(X) \rightarrow H^{2 *}(X, \mathbb{Q})$ is additive and it factors through the surjective map $K(X) \rightarrow \mathbb{Z} \oplus \mathrm{NS}(X) \oplus \mathbb{Z}$ given by $x \mapsto\left(\operatorname{rk} x, c_{1}(x), \chi(x)\right)$. This follows from the equality $\chi(x)=\int_{X} \operatorname{ch}_{2}(x)+$ $\operatorname{rk}(x)$ obtained from the Riemann-Roch theorem. Therefore $v(K(X))=$ $\mathbb{Z} \oplus H^{2}(X, \mathbb{Z})_{f} \oplus \frac{1}{2} \mathbb{Z} \rho_{X} \subset H^{2 *}(X, \mathbb{Q})$, where $H^{2}(X, \mathbb{Z})_{f}$ is the torsion free quotient of $H^{2}(X, \mathbb{Z})$.

On $H^{2 *}(X, \mathbb{Q})$ we introduce the Mukai pairing by $\langle x, y\rangle:=-\int_{X} x^{\vee} \wedge y$. Then the lattice $(v(K(X)),\langle\cdot, \cdot\rangle)$ is isometric to

$$
\left(\begin{array}{cc}
1 & 0 \\
0 & -1
\end{array}\right) \oplus\left(\begin{array}{cc}
0 & 1 \\
1 & 0
\end{array}\right) \oplus-E_{8}
$$

Note that the Mukai pairing induces on $H^{2}(X, \mathbb{Z})_{f}$ the intersection form $(\cdot, \cdot)$.

Definition 2.3. An element of $v(K(X))$ is called a Mukai vector. A Mukai vector $v$ is primitive if $v$ is primitive as an element of the lattice $v(K(X))$.

REMARK 2.4. A Mukai vector $v=2+c_{1}+t \varrho_{X}$ is not primitive if and only if $c_{1}$ is divisible by 2 and $t$ is odd. Indeed, $v$ can be divisible only by 2 and if it is divisible then we have the equality $2+c_{1}+t \varrho_{X}=2\left(1+c_{1}^{\prime}+\frac{1}{2} \rho_{X}+\frac{1}{2}\left(c_{1}^{\prime}\right)^{2}-c_{2}^{\prime}\right)$ for some $c_{1}^{\prime}$ and $c_{2}^{\prime}$. This is equivalent to $c_{1}=2 c_{1}^{\prime}$ and $t=1+\left(c_{1}^{\prime}\right)^{2}-2 c_{2}^{\prime}$.

Let us note that for a divisor $D$ we have $v\left(x \otimes\left[\mathscr{O}_{X}(D)\right]\right)=v(x) \exp (D)$, where $\exp (D)=1+D+\frac{1}{2} D^{2} \varrho_{X}$. Multiplication by $\exp (D)$ is an isometry of $(v(K(X)),\langle\rangle$,$) .$

In the case of Enriques surfaces the torsion free part of the Picard group is isomorphic to $H^{2}(X, \mathbb{Z})_{f}$. We also know that the lattice $\left(H^{2}(X, \mathbb{Z})_{f},(\cdot, \cdot)\right)$ is isometric to the orthogonal direct sum $\mathbb{H} \perp-E_{8}$, where $\mathbb{H}$ is a hyperbolic 
plane. The canonical basis of $\mathbb{H}$ is denoted by $\{\sigma, f\}$, so we have $\sigma^{2}=f^{2}=0$ and $(\sigma, f)=1$.

We will also use the following lemma which similarly to Remark 2.4 concerns divisors of $r, c_{1}$ and $s$ in a primitive Mukai vector.

Lemma 2.5. Let $v=r+c_{1}-(s / 2) \rho_{X}$ be a primitive Mukai vector. Then $\operatorname{gcd}\left(r, c_{1}, s\right)$ equals 1 or 2 . Moreover:

- if $\operatorname{gcd}\left(r, c_{1}, s\right)=1$ then either $r$ or $c_{1}$ is not divisible by 2 ,

- if $\operatorname{gcd}\left(r, c_{1}, s\right)=2$ then $c_{2}$ must be odd and $r+s \equiv 2 \bmod 4$.

Proof. If $\operatorname{gcd}\left(r, c_{1}, s\right)=1$ and $2 \mid \operatorname{gcd}\left(r, c_{1}\right)$ then $s=-r-c_{1}^{2}+2 c_{2}$ is even as well. If a prime number $p>3$ divides $\operatorname{gcd}\left(r, c_{1}, s\right)$ then $p$ divides $r, c_{1}$ and $c_{2}=\left(r+c_{1}^{2}+s\right) / 2$. This is also true for $p=2$ if we assume that $c_{2}$ is even. In both these cases $v=p v^{\prime}$ where $v^{\prime}$ is a Mukai vector associated to $r^{\prime}=r / p, c_{1}^{\prime}=c_{1} / p$ and $c_{2}^{\prime}=c_{2} / p+(p-1) c_{1}^{2} /\left(2 p^{2}\right)$. This follows from the equation

$$
\begin{aligned}
p\left(\frac{r^{\prime}}{2}+\frac{1}{2} c_{1}^{\prime 2}-c_{2}^{\prime}\right) & =p\left(\frac{r}{2 p}+\frac{1}{2 p^{2}} c_{1}^{2}-\frac{r+c_{1}^{2}+s}{2 p}+\frac{p-1}{2 p^{2}} c_{1}^{2}\right) \\
& =\frac{r}{2}+\frac{1}{2 p} c_{1}^{2}-\frac{r}{2}-\frac{1}{2} c_{1}^{2}-\frac{s}{2}+\frac{p-1}{2 p} c_{1}^{2}=-\frac{s}{2} .
\end{aligned}
$$

Therefore only 2 can divide $\operatorname{gcd}\left(r, c_{1}, s\right)$ but only if $c_{2}$ is odd. If $2 \mid \operatorname{gcd}\left(r, c_{1}, s\right)$ then $r+s \equiv r+c_{1}^{2}+s \equiv 2 c_{2} \equiv 2 \bmod 4$. It follows that $4 \nmid \operatorname{gcd}\left(r, c_{1}, s\right)$.

Corollary 2.6. Let $v=r+(r / 2) \delta+\xi-(s / 2) \rho_{X}$ be a primitive Mukai vector, where $r$ is even and $\delta \in H^{2}(X, \mathbb{Z})_{f}$ is primitive. Then $\operatorname{gcd}(r, \xi, s)$ equals 1 or 2 .

Proof. Let $p>2$ be a prime number such that $p \mid \operatorname{gcd}(r, \xi, s)$. Then $p \mid \operatorname{gcd}(r,(r / 2) \delta+\xi, s)$ which equals 1 or 2 . Suppose that $4 \mid \operatorname{gcd}(r, \xi, s)$. Then $\operatorname{gcd}(r,(r / 2) \delta+\xi, s)=2$ and by the above lemma $r+s \equiv 2 \bmod 4$, which leads to a contradiction.

2.3. Moduli spaces of sheaves of even rank on unnodal Enriques surfaces. Let $H$ be an ample divisor on $X$. Let $v=r+c_{1}-(s / 2) \varrho_{X} \in$ $H^{*}(X, \mathbb{Q})$ be a Mukai vector and let $L$ be a line bundle on $X$ such that $c_{1}(L)=c_{1}$. Then we denote by $M_{H}(v, L)$ the moduli space of Gieseker $H$-semistable sheaves with Mukai vector $v$ and with fixed determinant $L$. In the following, for a fixed Mukai vector $v, M_{H}(v, L)$ denotes $M_{H}(v, L)$ for some fixed line bundle $L$ satisfying $c_{1}(L)=c_{1}(v)$. This will not cause any problems since there are only two line bundles with the same first Chern class and they differ by a torsion line bundle so the corresponding moduli spaces are isomorphic.

For a complex variety $Y$ the cohomology with compact support has a natural mixed Hodge structure. This allows us to define the virtual Hodge 
number $e^{p, q}(Y)=\sum_{k}(-1)^{k} h^{p, q}\left(H_{c}^{k}(Y, \mathbb{Q})\right)$ and the virtual Hodge polynomial $e(Y)=\sum_{p, q} e^{p, q}(Y) x^{p} y^{q}$.

The moduli space $M_{H}(v, L)$ is constructed as the quotient of a certain open subset $Q$ of the Quot-scheme by an action of $\operatorname{GL}(V)$. Then the rational function

$$
e\left(M_{H}(v, L)\right)=e(Q) / e(\mathrm{GL}(V))
$$

is well defined and we call it the virtual Hodge polynomial of $M_{H}(v, L)$. It is known that for a general polarization $H$ it does not depend on the choice of $H$ (see [Yo, Proposition 4.1]).

In $\left[\mathrm{Yo}\right.$ it is shown that for an unnodal Enriques surface if $v=r+c_{1}-$ $(s / 2) \varrho_{X} \in H^{*}(X, \mathbb{Q})$ is a primitive Mukai vector such that $r$ is odd then the virtual Hodge polynomials $e\left(M_{H}(v, L)\right)$ and $e\left(\operatorname{Hilb}_{X}^{\left(\left\langle v^{2}\right\rangle+1\right) / 2}\right)$ are the same for general $H$. We want to obtain a similar result for even rank $r$.

The main ingredient of the proof of Yoshioka's theorem is the following proposition:

Proposition 2.7 (see [Yo, Proposition 4.5]). Let $X$ be an unnodal Enriques surface. Assume that $r, s>0$. If $c_{1}^{2}<0$ then for a general polarization $H$ we have $e\left(M_{H}\left(r+c_{1}-(s / 2) \varrho_{X}, L\right)\right)=e\left(M_{H}\left(s-c_{1}-(r / 2) \varrho_{X}, L^{\prime}\right)\right)$.

Note that for a Mukai vector $v=r+c_{1}-(s / 2) \varrho_{X}$ the condition $c_{1}^{2}<0$ is equivalent to $\left\langle v^{2}\right\rangle<r s$.

TheOREM 2.8. Let $X$ be an unnodal Enriques surface and let $v=r+c_{1}-$ $(s / 2) \varrho_{X} \in H^{*}(X, \mathbb{Q})$ be a primitive Mukai vector such that $r$ is even. Then for a general polarization $H$ we have the equality $e\left(M_{H}(v, L)\right)=e\left(M_{H}\left(r^{\prime}+\right.\right.$ $\left.\left.c_{1}^{\prime}-\left(s^{\prime} / 2\right) \varrho_{X}, L^{\prime}\right)\right)$, where $r^{\prime}$ is equal to either 2 or 4 .

Proof. To simplify notation we consider only the moduli spaces $M_{H}(v)$ without fixed determinant. This is sufficient since $M_{H}(v)$ consists of two disjoint isomorphic moduli spaces of type $M_{H}(v \cdot L)$.

We keep the notation from the previous subsection: $H^{2}(X, \mathbb{Z})_{f}=\mathbb{H} \perp$ $-E_{8}$ and the canonical basis of $\mathbb{H}$ is denoted by $\{\sigma, f\}$.

To prove the theorem first we deal with the following special case: $c_{1}=$ $(r / 2) b f+\xi$, where $b \in\{0,1,-1\}$ and $\xi \in-E_{8}$. Let us set $l=\operatorname{gcd}(r, \xi)$. By Lemma 2.1 we can find $\xi_{1} \in-E_{8}$ such that $\left(\xi+r \xi_{1}\right) / l$ is primitive and $s-2\left(\xi, \xi_{1}\right)-r\left(\xi_{1}^{2}\right)>\left\langle v^{2}\right\rangle$. Therefore replacing $v$ by $v \exp \left(\xi_{1}\right)$ we may assume that $\xi / l$ is primitive and $s>\left\langle v^{2}\right\rangle$. Since $v$ is primitive, by Corollary 2.6. $\operatorname{gcd}(l, s)$ is equal to either 1 or 2 . Since $\xi / l$ is primitive we have $\operatorname{gcd}(s, \xi)=$ $\operatorname{gcd}(l, s)$. Now by Proposition 2.7 we get

$$
e\left(M_{H}\left(r+c_{1}-(s / 2) \varrho_{X}\right)\right)=e\left(M_{H}\left(s-c_{1}-(r / 2) \varrho_{X}\right)\right) .
$$

Note that $s$ is even as $s+r=-2 \operatorname{ch}_{2}(v)$. Replacing $v=r+c_{1}-(s / 2) \varrho_{X}$ by $v^{\prime}=s-c_{1}-(r / 2) \varrho_{X}$, we may therefore assume that $r>\left\langle v^{2}\right\rangle$. By the 
above we may also assume that $l=\operatorname{gcd}(r, \xi)$ is equal to 1 or 2 , and $\xi / l$ is primitive.

Let us set $D=\sigma-\left(\eta^{2} / 2\right) f+\eta$, where $\eta$ is some element of $-E_{8}$ (note that $\eta^{2} / 2$ is an integer as $-E_{8}$ is an even lattice). Then $D^{2}=0$ and

$$
\left(c_{1}, D\right)=\frac{r}{2} b+(\xi, \eta) .
$$

Let us choose $\eta \in-E_{8}$ which satisfies the following conditions:

- $2(\eta, \xi)=s-2-r b$ if $l=2$ and $4 \mid s-r b-2$ or $l=1$,

- $2(\eta, \xi)=s-4-r b$ if $l=2$ and $4 \mid s-r b$.

Existence of such $\eta$ follows from Lemma 2.2 because $\xi / l$ is primitive and the above equalities are equivalent to $(\eta, \xi / l)=(s-2-r b) /(2 l)$ or $(s-4-r b) /(2 l)$, respectively.

Then we have

$v \exp (D)=r+\left(c_{1}+r D\right)+\frac{1}{2}\left(r D^{2}+2\left(c_{1}, D\right)-s\right) \rho_{X}=r+\left(c_{1}+r D\right)-\frac{\varepsilon}{2} \rho_{X}$,

where $\varepsilon$ is either 2 or 4 . Since $r>\left\langle v^{2}\right\rangle$ we can use Proposition 2.7 once again to obtain

$$
e\left(M_{H}(v)\right)=e\left(M_{H}(v \exp (D))\right)=e\left(M_{H}\left(\varepsilon-\left(c_{1}+r D\right)-\frac{r}{2} \rho_{X}\right)\right) .
$$

This proves the required assertion in this case.

Analogously exchanging $\sigma$ with $f$ we can deal with the case $c_{1}=$ $(r / 2) a \sigma+\xi$, where $a \in\{0,1,-1\}$ and $\xi \in-E_{8}$.

Now we use induction on $r$ to prove the theorem in the general case. This part is very similar to the second part of proof of [Yo, Theorem 4.6]. Let us write $c_{1}$ as $d_{1} \sigma+d_{2} f+\xi$ for some $\xi \in-E_{8}$. Replacing $v$ by $v \exp (k \sigma+l f)$ we can assume that $-r / 2<d_{1} \leq r / 2$ and $-r / 2<d_{2} \leq r / 2$. If $d_{1}$ is non-zero and $\left|d_{1}\right|<r / 2$ then following Yoshioka's proof we can reduce the assertion to lower rank and use the induction assumption. Similarly, we deal with the cases when $d_{2}$ is non-zero and $\left|d_{2}\right|<r / 2$. So the only cases that we are left with are when the pair $\left(d_{1}, d_{2}\right)$ is equal to $(0,0),(0, r / 2),(r / 2,0)$ or $(r / 2, r / 2)$. But we already proved the theorem in three of these cases and the only case left is $\left(d_{1}, d_{2}\right)=(r / 2, r / 2)$.

In this case we have $c_{1}=(r / 2) \sigma+(r / 2) f+\xi$ for some $\xi \in-E_{8}$. To deal with this case we need to consider another orthogonal decomposition of the lattice $H^{2}(X, \mathbb{Z})_{f}$. Namely, if $\left\{e_{1}, \ldots, e_{8}\right\}$ denotes the canonical basis of $-E_{8}$ then we set $\sigma^{\prime}=\sigma, f^{\prime}=\sigma+f+e_{1}, e_{1}^{\prime}=e_{1}+2 f$ and $e_{i}^{\prime}=e_{i}$ for $i=2, \ldots, 8$. Then $\mathbb{H}^{\prime}=\mathbb{Z} \sigma^{\prime} \oplus \mathbb{Z} f^{\prime}$ is a hyperbolic plane and its orthogonal complement in $H^{2}(X, \mathbb{Z})_{f}$ is isometric to $-E_{8}$ with canonical basis $\left\{e_{1}^{\prime}, \ldots, e_{8}^{\prime}\right\}$. Let us write $c_{1}$ in this new decomposition as $a \sigma^{\prime}+b f^{\prime}+\xi^{\prime}$ for $\xi^{\prime} \in-E_{8}$. Comparing 
the coefficients at $\sigma$ we see that $a+b=r / 2$. This reduces the problem to the case already considered.

Similar but much simpler considerations lead to another proof of the following reformulation of Kim's theorem:

Theorem 2.9 ([Kim1, Theorem]). Let $v=2+c_{1}+t \varrho_{X} \in H^{*}(X, \mathbb{Q})$ be a rank 2 Mukai vector. Then there exists a divisor $D$ such that for some $c_{1}^{\prime} \in H^{2}(X, \mathbb{Z})_{f}$ we have $v \exp (D)=2+c_{1}^{\prime}+0 \varrho_{X}$ or $v \exp (D)=2+c_{1}^{\prime}+\varrho_{X}$.

\section{References}

[BPS] G. Banica, M. Putinar und C. Schumacher, Variation der globalen Ext in Deformationen kompakter komplexer Räume, Math. Ann. 250 (1980), 135-155.

[BHPV] W. Barth, K. Hulek, Ch. Peters and A. Van de Ven, Compact Complex Surfaces, 2nd ed., Ergeb. Math. Grenzgeb. 4, Springer, Berlin, 2004.

[CD] F. Cossec and I. Dolgachev, Enriques Surfaces. I, Progr. Math. 76, Birkhäuser Boston, Boston, MA, 1989.

[EGA] A. Grothendieck, Éléments de géométrie algébrique III. Étude cohomologique des faisceaux cohérents. II, Inst. Hautes Études Sci. Publ. Math. 17 (1963), 5-91.

[HL] D. Huybrechts and M. Lehn, The Geometry of Moduli Spaces of Sheaves, Aspects Math. E31, Vieweg, Braunschweig, 1997.

[Kim1] H. Kim, Stable vector bundles on Enriques surfaces, PhD thesis, Univ. of Michigan, 1990.

[Kim1] —, Moduli spaces of bundles mod Picard groups on some elliptic surfaces, Bull. Korean Math. Soc. 35 (1998), 119-125.

[Kim2] -, Moduli spaces of stable vector bundles on Enriques surfaces, Nagoya Math. J. 150 (1998), 85-94.

[Kim3] -, Stable vector bundles of rank two on Enriques surfaces, J. Korean Math. Soc. 43 (2006), 765-782.

[La] A. Langer, Lectures on torsion-free sheaves and their moduli, in: Algebraic Cycles, Sheaves, Shtukas, and Moduli, Trends Math., Birkhäuser, Basel, 2008, 69-103.

[OV] C. Okonek and A. Van de Ven, Stable bundles and differentiable structures on certain elliptic surfaces, Invent. Math. 86 (1986), 357-370.

[Yo] K. Yoshioka, Twisted stability and Fourier-Mukai transform I, Compos. Math. 138 (2003), 261-288.

Marcin Hauzer

Institute of Mathematics

Warsaw University

Banacha 2

02-097 Warszawa, Poland 
